Results 150 patients between 60 and 91 years old (mean 69.37 and $\mathrm{SD} \pm 7169)$ participated. Most patients were women, white race, widowhood, low educational level and family income. 54\% was considered reasonable general health, $14.7 \%$ poor and $51.3 \%$ the same condition as last year. $66 \%$ were edentulous. Highlighted problems: tartar, inflamed gums, cavities, residual root, soft tissue injuries, prosthesis needs. Last year, only $20 \%$ visited the dentist. Main cited diseases: vision, hypertension, hearing, osteoporosis, diabetes mellitus, cardiovascular diseases, rheumatism and cholesterol. $10.7 \%$ were smokers and $69.3 \%$ used drugs. Symptoms of mild and severe depression were detected. Statistical association verified among depression symptoms with: perceived general health $(p=0.003)$, osteoporosis $(p=0.007)$. Depression and oral health: smoking ( $p=0.040)$, dry mouth $(p=0.001)$, burning mouth $(p=0.055)$, pain in the mouth $(p=0.009)$, swelling of the mouth $(p=0.001)$ and using prosthesis $(p=0.005)$.

Conclusion Older people's oral health is poor and most show depressive symptoms. The consequences of teeth condition were significant with depressive problems, providing public health burden that must be considered by public policy.

\section{SP3-61 PRESENT AND PREVIOUS ADOLESCENT PREGNANCY: SOCIOECONOMIC CHARACTERISTICS AND THE EFFECT ON NEWBORN WEIGHT}

doi:10.1136/jech.2011.1429760.61

S Gama, ${ }^{*}$ A P Esteves-Pereira, M do Carmo Leal. ENSP, FIOCRUZ, Rio de Janeiro, Brazil

Introduction The negative effects of pregnancy in adolescence on maternal and child health have been described. However gaps in the literature examination the association between this exposure and outcome remain. The aim of this study was to examine the association between socioeconomic characteristics of three groups of mothers (adolescents ( $<20$ years old), women $\geq 20$ years old with a previous pregnancy during adolescence and women $\geq 20$ years old without a pregnancy during adolescence) and low birth weight $(<2500 \mathrm{~g})$.

Methods 1681 women in the first trimester of pregnancy were interviewed in two cities; follow-up at postpartum was available for 1540. To verify the hypothesis of homogeneity of proportions, $\chi^{2}$ tests were used. To verify the association with low birth weight we conducted crude and adjusted logistic regression analyses with a significance level of 0.05 .

Results Women $\geq 20$ years old with a history of pregnancy in adolescence were more socioeconomically deprived than other groups. Compared to women $\geq 20$ years old without a history of pregnancy in adolescence, women $\geq 20$ years old with a history of pregnancy in adolescence had a lower level of educational attainment [OR 2.68], lower social status [OR 1.96] and lower income [OR 2.71]. Low birth weight was greater in the children of adolescent mothers [OR 1.36]. Conclusions These findings suggest that in Brazil adolescent pregnancy is a social problem and women with a pregnancy in adolescence are socioeconomically disadvantaged through their lives.

\section{SP3-62 AGREEMENT BETWEEN MEDICAL RECORD AND PARENT REPORT FOR EVALUATION OF CHILDHOOD FEBRILE SEIZURES}

doi:10.1136/jech.2011.1429760.62

B Ackerson, ${ }^{*}$ L S Sy, J F Yao, T C Cheetham, T L Jones, S J Jacobsen. Kaiser Permanente Southern California, Pasadena, California, USA

Introduction Little is known about the quality of medical record data used to evaluate febrile seizures despite its influence on vaccine recommendations.
Methods Parent report from phone interviews and mailed questionnaires was compared to abstracted medical records of 110 children with febrile seizures between ages 3 and 60 months Concordance between parent report and medical records for characteristics and predisposing factors of febrile seizures was assessed by per cent total agreement and $\kappa$ statistic. Sensitivity of medical record and parent report was determined setting parent report and medical record as the standard, respectively (Tisnado, 2006).

Results Per cent total agreement between medical record and parent report was good for many variables studied $(62.5 \%$ had $>70 \%$ agreement). However, $\kappa$ was low for all measures $(100 \%$ had $\kappa$ $<0.33)$ For seizure characteristics, parent report was more sensitive than medical record (69.9\% (95\% CI 50.0 to 88.9$)$ and $31.9 \%(95 \%$ CI 18.2 to 45.7), respectively) while both were sensitive for fever (91.0\% (95\% CI 85.4 to 96.6 ) vs $91.9 \%(95 \%$ CI 86.6 to 97.3$)$ respectively). Neither was sensitive for predisposing factors $(62.1 \%$ (95\% CI 41.5 to 81.8$)$ ) and $64.1 \%$ (95\% CI 44.0 to 83.3), respectively). Conclusion These data demonstrate the limitations of using only medical records or parent report to assess febrile seizures. Collection of information from both sources is required to most accurately portray the spectrum of predisposing factors and seizure characteristics of childhood febrile seizures.

\section{SP3-63 FROM PRE-NATAL TO 3 MONTHS OLD: LONGITUDINAL STUDY WITH ADOLESCENT MOTHERS}

doi:10.1136/jech.2011.1429760.63

S Gama, ${ }^{*}$ A P Esteves-Pereira, M do Carmo Leal. ENSP, FIOCRUZ, Rio de Janeiro, Brazil

Introduction The negative effects of pregnancy in adolescence on the mother and child health has been well established, however there are still remaining gaps to more studies to explore this cause-effect relation

Objectives To compare three groups of mothers-adolescents $(<20$ years) and two composed of $\geq 20$-year-old women classified according to past experience of pregnancy during adolescence-with respect to perinatal care.

Methods A sample of 1681 women was interviewed in two cities during the first trimester of pregnancy, a follow-up sample of 1540 at postpartum and 1434 after 3 months. We conducted crude and adjusted logistic regression analysis for each outcome, with a significance level of $5 \%$

Results The most disadvantaged conditions were found among the adolescent's mothers and those with a history of pregnancy in adolescence. In comparison with women who hadn't been pregnant in adolescence, they had, respectively, a higher frequency of inadequate pre-natal-care [OR 1.8 and 1.5], no postpartum visit [OR 1.3 and 1.5], no performance of newborn screening test [OR 2.3 and 2.1] and baby's first doctor visit $>1$-month-old [OR 1.7 and 1.8]. No differences were found regarding breastfeeding at 3-month-old and immunisation. After adjustment for city, educational level and skin colour the association remained statistically significant for pre-natal inadequacy [OR 1.7 and 1.4] and baby's first doctor visit $>1$-monthold [OR 1.5 and 1.6].

Conclusions Previous and current adolescence pregnancy seems to be a risk factor for inadequate healthcare for both women and its offspring.

\section{SP3-64 ASSOCIATION BETWEEN HISTORY OF TUBERCULOSIS AND VEGETARIANISM FROM A NATIONALLY REPRESENTATIVE SURVEY IN INDIA}

doi:10.1136/jech.2011.142976o.64

${ }^{1} \mathrm{P}$ Arora, ${ }^{1} \mathrm{P}$ Jha, ${ }^{1} \mathrm{~N}$ Nagelkerke. ${ }^{1}$ Centre for Global Health Research (CGHR), Li Ka Shing Knowledge Institute, St. Michael's Hospital, Dalla Lana School of Public Health, 
Toronto, Ontario, Canada; ${ }^{2}$ Department of Community Medicine, United Arab Emirates University, Al-Ain, United Arab Emirates; ${ }^{3}$ Department of Public Health, Erasmus Medical Center, Rotterdam, The Netherlands

Introduction Infection with $M$ tuberculosis is a necessary but not sufficient condition for development of clinical Tuberculosis (TB). The reasons why some tuberculosis infections progress to clinical $\mathrm{TB}$ while most remain latent is not clear. A vegetarian diet has been implicated as a risk factor for tuberculosis among South-Asians in the UK.

Methods To explore whether this is the case in India we analysed data from the nationally representative National Family Health Survey-3 (2006) which collected information on TB and diet, and tested for HIV-1. Tuberculosis was reported by heads of households. Results Vegetarianism was not a risk factor for tuberculosis among HIV-1 negative married men and women between 15 and 49 (women) or 54 (men) years [OR 0.66 (95\% CI 0.49 to 0.89)] while poverty and a history of blood transfusions were. Individuals reporting TB were slightly older than those who did not (31.8 vs 29.4 in women, 39.3 vs 37.2 in men). Vegetarians were also slightly older than non-vegetarians (29.5 vs 29.4 in women, and 37.9 vs 37.0 in men). Except for 11 men and 11 women all individuals with TB were reported to have received treatment for their condition. Urban residence, poverty, higher age, and blood transfusion were positively associated with TB

Conclusion Vegetarianism appeared to be protective against TB, perhaps due to confounding by unmeasured life-style factors. As it seems unlikely that confounding has masked a strong positive association between vegetarianism and $\mathrm{TB}$, we conclude that vegetarianism is not a risk factor for TB in India.

\section{SP3-65 MISSION IMPOSSIBLE? EVALUATING THE EFFECTIVENESS OF HIGHLY RADICAL CANCER SURGERY WITH INSIGHTS FROM A PROSPECTIVE STUDY OF PELVIC EXENTERATION}

doi:10.1136/jech.2011.1429760.65

1,2 J Young, ${ }^{*} 1,2 \mathrm{~L}$ Rodwell, ${ }^{3} \mathrm{~A}$ Heriot, ${ }^{1,2} \mathrm{M}$ Solomon. ${ }^{1}$ University of Sydney, Sydney, New South Wales, Australia; ${ }^{2}$ Sydney Local Health Network, Sydney, New South Wales, Australia; ${ }^{3}$ Peter MacCallum Cancer Centre, Melbourne, Victoria, Australia

Introduction Policy makers, clinicians and patients need robust evidence of the effectiveness of healthcare interventions to inform decision-making. Highly aggressive cancer surgery has major implications for patients and requires substantial healthcare resources, but presents considerable challenges for evaluation. Although a randomised controlled trial is the optimal study design to investigate therapeutic effectiveness, randomisation is not possible where there are major, irreversible, differences in the treatments in intervention and control groups. Furthermore, with novel surgical techniques, as experience is gained there may be changes in patient selection, referral patterns and outcomes over time, introducing additional potential sources of bias. These methodological issues will be explored using data from a ongoing prospective, multi-centre evaluation of pelvic exenteration, a highly radical procedure for pelvic cancer where the alternative is palliative care.

Methods Baseline demographic, clinical and quality of life data are collected during the period of treatment decision-making, with clinical and quality of life outcomes assessed every 3 months thereafter. Trends in referral patterns, treatment choices, patient characteristics and outcomes will be compared between groups.

Results Since 2008, 144 patients have been assessed, with 111 (77\%) undergoing pelvic exenteration. Although the absolute rate of patient referral has increased over time, there is no significant trend in the proportion undergoing surgery $\left(\chi^{2}{ }_{1}=0.46, p=0.5\right)$. Trends in group characteristics and outcomes to June 2011 will be presented.
Conclusion Non-randomised evaluations of complex surgery are challenging but necessary. Ensuring the comparability of treatment groups is critical for robust evaluation.

\section{SP3-66 CARIES IN CHILDREN ON THE EAST SIDE OF MEXICO CITY STUDY}

doi:10.1136/jech.2011.1429760.66

L P S Martínez, * J M Núñez Martínez, C Osorno-Escareño Mdel, L Romero, U Érika Cenoz. Metropolitan Autonomous University Xochimilco, Distrito Federal, Mexico

Introduction The fluor addition in salt began in Mexico at the early '90s; consequently there has been a decrease of caries cases, but with different average results at the age of 12 in the whole country. Mexico City is one of the zones that present a moderate average (2.1-3). Considering this information it is pertinent to expose the benefit of fluoridated salt in different zones of Mexico City, as well as the different marginalisation characteristics.

Objective To know the experience of caries in children on the East side of Mexico City.

Methods Cross and observational survey. Informed previous consent, 552 students were analysed from the area of Iztapalapa on the East side of Mexico City. This zone is rated as one of the most marginalised ones of the federative entity. Previous calibration of two examiners, both caries dentitions were diagnosed (WHO). Data analysed with SPSS, getting central tendency results and dispersion of gender, age and caries variables. In order to distinguish homogeneity of variables it was used $\chi^{2}(p<0.05)$.

Results 285 girls (52\%) and 267 boys (48\%) were examined, average age of 8.65 years old. The global dmft was $3.79+3.48$ and the global DMFT was $1.67+2.07$. There was no significant gender difference in both dentitions, but there was one related to age $(p<0.0001)$. The average dmft- 6 was $4.98+4.10$ and the DMFT-12 was $3.22+2.71$.

Conclusions The studied population presented high rates of caries at the age of twelve, a situation that suggests major efforts in mouth health prevention

\section{SP3-67 KAPOSI SARCOMA INCIDENCE IN UGANDA AND ZIMBABWE, BEFORE AND DURING HIV/AIDS EPIDEMIC}

doi:10.1136/jech.2011.1429760.67

${ }^{1} \mathrm{~K}$ Chaabna, ${ }^{*} \mathrm{~F}$ Bray, ${ }^{2} \mathrm{H}$ Wabinga, ${ }^{3} \mathrm{E}$ Chokunonga, ${ }^{4,5} \mathrm{P}$ Vanhems, ${ }^{1} \mathrm{D}$ Forman ${ }^{1}$ International Agency for Research on Cancer, Lyon, France; ${ }^{2}$ Kampala Cancer registry, Department of Pathology, Makerere University, Kampala, Uganda; ${ }^{3} Z i m b a b w e ~ C a n c e r$ registry, Parirenyatwa Hospital, Harare, Zimbabwe; ${ }^{4}$ Service d'Hygiène, Epidémiologie et Prévention, Hôpital Edouard Herriot, Hospices Civils de Lyon, Lyon, France ${ }^{5}$ Laboratoire d'Epidémiologie et de Santé Publique, CNRS, UMR 5558, Université de Lyon, Université Lyon 1, Lyon, France

Introduction We described Kaposi sarcoma (KS) incidence changes during the AIDS emergence and subsequently in Zimbabwe with a high prevalence of HIV infection and Uganda with an intermediate HIV/AIDS prevalence and known to have high rates of endemic KS. Methods Cancer data were extracted from the cancer registries of Harare (1990-2005), Bulawayo (1963-1972), Zimbabwe and Kyadondo, Uganda (1960-1971 and 1991-2007). We used a join point model to analyse the time trends of age-standardised rates, and the populations were compared by computing the standardised incidence ratio and $95 \%$ CIs.

Results In both countries, an increase in the incidence of $\mathrm{KS}$ accompanied the AIDS emergence. In Harare, KS incidence seemed to change according to the time trend of HIV/AIDS prevalence; however, in Kyadondo, we observed an increase of KS incidence in people over 50 years despite the decrease in HIV prevalence. Before 